\title{
Forming Computational Thinking and Computer Modeling Project Activities in the Physics Course of the Technical University
}

\author{
Alexander $V$. Baranov $^{1 *}$ \\ ${ }^{1}$ Novosibirsk State Technical University NETI, Novosibirsk, 630073, Russia
}

\begin{abstract}
The article discusses contextual technology of teaching and learning physics of IT students at the Technical University. The main goal of this research is development and analysis contextual technology to maintain and increase IT students' interest in physics with parallel formation of computational thinking and competencies in computer science applications. This technology takes place in several contexts: the scientific method and modeling of physical processes, computational thinking and professional competence. The all contexts are present in four stages of the training cycle: lectures, seminars, lab works, and computer modeling of physical processes. As an example we demonstrate using the contextual technology for the physics course topic "Dynamics of a rigid body rotational motion" in all four stages of the training cycle. The example of student's team development of the virtual lab work "Precession and nutation of a gyroscope" is given. According to the Novosibirsk State Technical University professors and students, the author's technology has demonstrated its effectiveness in the formation of professional thinking and competencies of the IT students in teaching physics at the Technical University.
\end{abstract}

\section{Introduction}

The development of students' professional thinking and core competencies are the most important tasks and outcomes of higher education.

For graduates of technical universities, the necessary components of thinking and competencies are largely determined by progress in science, technique and technology. The significant changes have occurred in the structure of the world higher education since it was beginning the era of Information Technologies (IT). In addition to traditional engineering specialties, new IT specializations appeared at the technical universities: computer science, computer engineering, and information technology.

And todays the Information Society transition to the stage of the Fourth Industrial Revolution (Schwab, 2017) opens up new opportunities and poses new problems in teaching IT students. Industry 4.0 gives rise to a number of challenges for IT: development

\footnotetext{
* Corresponding author: baranov@ corp.nstu.ru
} 
of cyber-physical systems (CPS), implementation of the concept of smart cities, Big Data analysis, quantum computing, and artificial intelligence.

Professional experts predict that CPS technologies will be Industry 4.0 basis, and the CPS revolution will be more transformative for the Information Society than the IT revolution of the past four decades (Lee, 2015; Müller, 2017). According to the experts, a large number of new specializations in the field of science, technical and technological applications of IT and CPS are expected.

By means of the CPS technology our society is involved in a very fast changing world. This fast change must be reflected into the educational system of course. And the most significant interest now is shown to the training of IT specialists in the field of the CPS. For example, the National Science Foundation of USA recently sponsored a multiyear study by the National Academies of Sciences, Engineering, and Medicine to develop clearly articulated criteria for curricula to address effective CPS education (Stankovic, Sturges \& Eisenberg, 2017).

The expected expansion of the IT specialties list and a significant increase in the requirements for IT professionals necessitate fundamental changes in the educational practice of the technical universities that implement the training in IT and CPS applications. Industry 4.0 poses new challenges for Education 4.0 (Wallner \& Wagner, 2016; Chea \& Huan, 2019). To be successive in Industry 4.0 students have to learn how to apply their skills in shorter terms to a variety of situations and they should be acquainted with project based learning before their work life begins (Chea \& Huan, 2019).

We think Education 4.0 requires the earliest possible start an active formation of professional thinking and competencies of future IT specialists for Industry 4.0. So the important problem now is beginning this formation already at the early stage of education at the technical universities - when IT students study the general education disciplines.

For the IT students physics is one of the general education disciplines. And for IT students specializing in the field of CPS, physics can and have to become one of the main academic courses because CPS is "an orchestration of computers and physical systems" (Lee, 2015). As academic discipline, physics has a great didactic potential (Redish, 1999). In teaching IT students, this didactic potential should be used as efficiently as possible, given increased interest of students in IT and low motivation for studying physics. This potential should work for the future, fostering thinking and competencies of IT students for Industry 4.0.

And so that is our main question: is it possible, by teaching physics, to start developing professional thinking and competencies of IT students at an early stage of education at the technical universities?

The positive answer is given by the theory of contextual teaching and learning. As Noah Finkelstein showed in his study of the context role in teaching physics, "students (and other educational participants) shape and are shaped by the context in which these educational endeavours occur" (Finkelstein, 2005).

Our research shows that IT students' professional thinking and competencies formation in the process of studying physics is possible. But it requires a special contextual approach to the educational process using concepts of Scientific method, Modeling, Problem and Project based teaching and learning technique. It has to be contextual teaching and learning physics (CTLPh) technology of IT students for the Industry 4.0.

The author has developed, used and researched the CTLPh technology at the Faculty of Applied Mathematics and Computer Science of the Novosibirsk State University NETI (NSTU NETI). 


\section{Theoretical framework}

With the advent of computers and algorithmic languages, programming skills were proclaimed as the second literacy (Ershov, 1981). And the awareness of computer's didactic opportunities led to the idea of developing a special style of thinking computational thinking (CT) (Papert, 1980; DiSessa, 2000). Seymour Papert may have been the first to use the term "CT" in 1980, when in his famous book he described a mental skill children develop from practicing programming (Papert, 1980). But in fact the emergence of number ideas about $\mathrm{CT}$ has a long history dating back to the fifties of the last century (Tedre \& Denning, 2016; Denning, 2017).

The modern discussion and interpretation of CT began in 2006 with an essay by Jeannette Wing (Wing, 2006). Wing's main idea was that everyone would benefit from learning to think like a computer scientist. "However, the movement has been criticized for vagueness, ambiguous definitions and visions of CT, and arrogance, as well as for bold, unsubstantiated claims about the universal benefit of CT" (Tedre \& Denning, 2016). But it's obvious now that CT concept is very useful for teaching and learning of IT students (Denning, 2017).

In our research we use the Alfred Aho definition of CT: "We consider computational thinking to be the thought processes involved in formulating problems so their solutions can be represented as computational steps and algorithms" (Aho, 2012). CT is a base of professional thinking of IT specialists.

To form the CTLPh technology we outline some components of CT that can be used in all steps of training (Hsu, Chang, Hung, 2018):

- Abstraction.

- Algorithm design.

- Data analysis and representation.

- Decomposition.

- Pattern generalization and recognition.

- Connection to other fields.

- Modeling.

- Visualization.

- Simulation.

In a number of papers, the important idea was expressed that integration of computer modeling and simulation into the physics course can provide significant support for the development of conceptual understanding (Wilson \& Redish, 1989; Gould \& Tobochnik, 2001; Landau, 2006; Chabay \& Sherwood, 2008, De Jong, Linn \& Zacharia. 2013).

In 2008 Marc Prensky formed the concept that students can be designers and creators of educational computer games (Prensky, 2008). In the CTLPh technology we realize analog of that idea in organization of IT students' project activity of computer modeling physical systems and processes for virtual labs.

\section{Method}

Before talking about the CTLPh technology, it is necessary to define the term "context", although it is considered quite multivalued. One of the generalized definitions is given in the dictionary by Merriam-Webster: «Context now most commonly refers to the environment or setting in which something (whether words or events) exists. When we say that something is contextualized, we mean that it is placed in an appropriate setting, one in which it may be properly considered» (Merriam-Webster, 2019). 
Edward Redish explicitly lists the context as one of five principles in education: “...the context principle: What people construct (for mental models) depends on the context including their mental state" (Redish, 1999).

In the CTLPh technology we use the psychological context definition that was given in 1987 by A. A. Verbitsky as "a system of interior and exterior factors and conditions of human behavior and activity, which influence the peculiarities of perception, understanding and transformation of some concrete situation and which determine the meaning and sense of the situation as a whole and the components it comprises" (Verbitsky \& Kalashnikov, 2013).

For the author, the main goal is to develop the CTLPh technology that allows forming computational thinking and competencies of IT students at the early stage of training when studying physics. High motivation of IT students to master IT acts as an internal factor contributing to this formation. As an external factor will be a special organization of the physics course with contextual emphasis on the substantive aspects of IT.

The conceptual basis of training in the CTLPh is the scientific method and modeling as one of its main components (Johnson-Laird,1983; Halloun, 2004; Hestenes, 2006; Etkina, Gentile \& Warren, 2006; Zwickl, Hu, Finkelstein \& Lewandowski, 2015). The concept of experiential learning cycle of David Kolb (Kolb, 1971) and insights from Experiential Learning Theory (Kolb, 2015) were used to organize the conceptual CTLPh technology cycle (see Fig.1). In accordance with this cycle, educational activities of the IT students are organized.

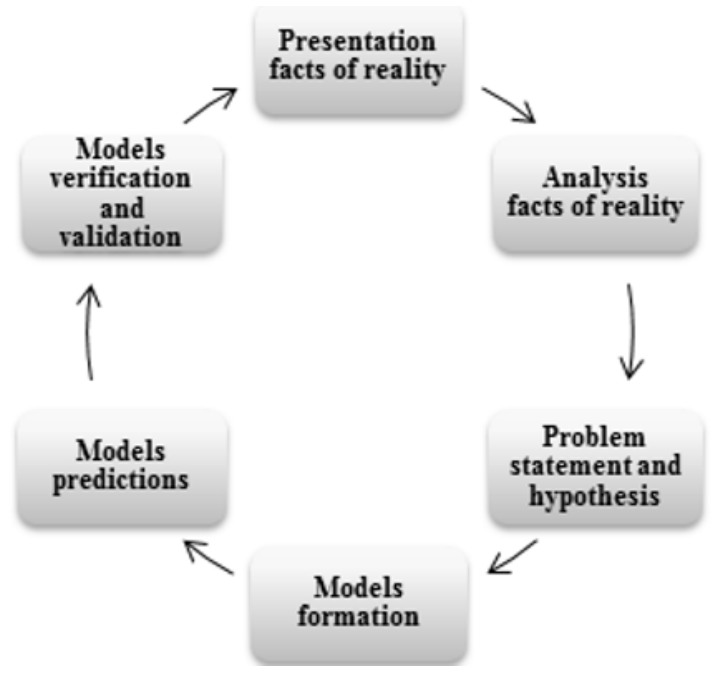

Fig.1. The CTLPh technology cycle

For every theme of physics course the CTLPh cycle is used multiple times: it is present at every stage of training and combines all the four stages: lecture, seminar, laboratory work, and computer modeling of physical systems and processes.

\subsection{Lectures in the CTLPh technology}

Based on the CTLPh cycle, the following concepts are emphasized in lectures in the context of formation the CT:

- Abstraction is demonstrated when introducing physical theory concepts, constructing mathematical models of physical systems, interactions and processes.

- Decomposition is demonstrated when analyzing structures and processes. 
- $\quad$ Algorithmization is demonstrated in formation and using of mathematical models. Some universal algorithms are demonstrated for solving certain classes of physical problems.

- Generalization is demonstrated when analyzing facts of physical reality and passing to hypotheses, analyzing algorithms, comparing model predictions and experimental results.

Thus, in the lectures, along with presentation of the content of physics, there is a contextual and explicit reference to the content of the CT components with the emphasis on them.

\subsection{Seminars in the CTLPh technology}

The transition from a lecture to a seminar aims to train the IT students to work with conceptual and mathematical models of physical reality.

- Abstraction. In the seminar's problem tasks some real objects usually appear in terms of analyzing physical situation. After discussing the problem, students are invited to make the transition from physical reality (referred to in the task) to hypothesis, abstract and mathematical models. In this procedure, certain simplifications are used, which often make it possible to obtain analytical mathematical solutions.

After receiving an analytical solution, we discus some transitions to more complex models, which mathematical analysis is possible only using numerical algorithms.

- Decomposition. Decomposition in solving physical problems is associated with the speculative partitioning of the analyzed reality into components and stages. This implies, for example, identifying systems and analyzing properties of interacting bodies and fields' distribution, highlighting the sequence of processes and determining the physical laws applicable to their description. Decomposition is required when analyzing bodies' movement in fields: first, the students solve the problem of fields' distribution in space, and then they solve the problem of bodies' movement in the fields.

Special attention is drawn to possible problem situations when a search for selfconsistent solutions is required due to the influence of moving bodies on the field's spatial distribution.

- Algorithmization. Algorithm, as a certain sequence of actions in solving problems, includes the two components described above, which in the process of analysis lead to mathematical formalization and the appearance of characteristic equations. The solution of the latter involves the knowledge of certain methods and techniques of mathematics.

In our discussion of methods for the considered problems, we demonstrate some examples of conditions' modification requiring a transition to numerical methods and algorithms.

Teaching the IT students to solve problems, we demonstrate important facts of possible branching in the processes of forming algorithms: in our reasoning, arguments and implementation of algorithms, either directly or implicitly, there are logical constructions like "if - then", "if - then - else" and others. They relate to the definition of conceptual models, abstraction, decomposition, justification of the applicability of laws, and the search for methods of solving mathematical equations. After solving some proposed problem tasks, the students discuss the implemented algorithm and its formalization.

- Generalization. Generalization in solving educational problems is associated with analysis, comparison and identification of analogies in different proposed physical 
situations. Analysis of the conditions and solutions of several problems can lead the students to identify universal algorithms for certain classes of problems. For this purpose, several problems of different content are selected. And the students look for common meanings in different initial contents and formalize algorithms. At the seminars on the lectures' topics, some methods of presenting information that have already been considered are used. One of these ways is "frameworks" symbolic formalizations of universal algorithms for solving specific classes of physical problems. To prepare students for practical exercises some specialized manuals are used. For every seminar the manuals include: training topics, training questions, description of typical algorithms, task sets, bibliography.

\subsection{Laboratory works in the CTLPh technology}

In the CTLPh laboratory experiments are positioned as a means of verifying theoretical concepts, models, lows and principles of physics, which were discussed at the lectures and seminars.

Before doing any lab work, the students have to demonstrate their understanding of the goal of a particular experiment in the context: theoretical ideas and how we can verify them in experiments.

After completing a lab work, the students report the results of experiments in the form of tables, graphs and prints of photographs taken from smartphones. Every report ends with conclusions in which a comparative analysis of the theoretical concepts and the experimental results is carried out.

The specialized training manuals of two types we use for the lab works. The first type of manuals contains descriptions of the physical experiments and instructions for performing the lab works. For every lab work, a specific goal is set and the required tasks are formulated. The second type manuals contain a three-level system of questions on the topics of the lab works.

The first level is a test of knowledge of definitions of abstract concepts, models and formulations of physical laws. The second level is a test of using physical concepts, models and laws to analyze and predict the results of laboratory experiments. The third level is a test of using physical concepts and laws to analyze proposed physical problem tasks.

The first level can be checked using electronic means (for example, LMS Moodle). But the second and the third levels are checked only in a conversation.

\subsection{Computer modeling in the CTLPh technology}

Computer modeling is the final stage of the CTLPh technology for the IT students.

Upon completion of this final stage, diagnostics of the level of formation of the CT components and the selected competencies determining the future professional activity of the IT students takes place.

Only experimental IT students' groups take part in the CTLPh computer modeling project activities. The students of those groups form the teams of 3-4 people. The every team works on its project related to computer modeling of physical systems and processes.

The students' work on the project is complete with the development of a software product that allows for virtual physical experiments. And some of the students' software products are interactive physical virtual lab works.

The students' participation in such project activities allows you to check the degree of formation their computational thinking and to lay foundations of the series of professional competencies. 
The process of the project computer modeling activity requires decision a number of problems (Baranov, 2016, 2017):

1. Formation of conceptual physical models.

2. Mathematical formalization of models and choice of methods.

3. Definition of the graphic interface structure and 3D image design of the modeled systems and process.

4. Specification of the structure and content of the software algorithm.

5. Creation of virtual 3D models using graphical editors.

6. Development and debugging the software product.

7. Virtual experiments.

8. Reporting and presentation.

The IT students' teams solve a number of problems related to the different education disciplines - physics, mathematics, programming, design. And the teams work on the projects independently implementing all the stages of creating software products.

For their program realizations the IT students use: high-level languages $\mathrm{C}++$ and $\mathrm{C} \#$; program platforms Visual Studio, Qt Creator and NET Framework; open-source graphics tools Blender and OpenGL. The 3D dynamic graphics in conjunction with interactive interfaces allow the students' virtual labs to visualize and explore various aspects related to the simulated physical systems and processes.

The students' software products can be used in the e-learning environment well complementing existing traditional didactic means (Baranov, 2018). So, the IT students really can be designers and creators of educational resources (Prensky, 2008).

\section{Examples of using the CTLPh technology}

As an example we demonstrate using the CTLPh technology for the physics course topic "Dynamics of a rigid body rotational motion". The topic is quite difficult for students to understand (Rimoldini \& Singh, 2005). This is particularly due mathematical abstractions pseudovectors (angular momentum and torque) and tensor (inertia tensor) (Rao, 2006). Using CTLPh technology cycle allows us to logically justify these abstract concepts.

\subsection{Lecture "Dynamics of a rigid body rotational motion"}

Presentation facts of reality. At first the students' audience is offered some demonstrations of real or video experiments with rigid body rotation.

Analysis facts of reality. When analyzing experiments, the students pay attention to the peculiarities of the bodies' reaction to the force impact during rotational motion.

Problem statement and hypotheses. These experiments raise a number of problem questions, for example:

1. Why is it most effective to untwist or stop a rotating disk if the force is applied far from the rotation axis and is directed tangentially?

2. Why, with the same masses and the same radii, is it easier to untwist a solid disk than a wheel with spokes?

The students put forward hypotheses:

1. For the rotational motion, the force impact degree is determined not only by force, but also by the point of its application.

2. For the rotational motion, the inertia of a rigid body is determined not by body mass, but by its distribution relative to the axis.

Models formation. The conceptual physical and mathematical models are formed to describe the rotational motion dynamics. From the considered real facts analysis and the 
proposed hypotheses, the necessity of introducing new abstract constructs is logically demonstrated.

Using three well known vectors (radius vector $\boldsymbol{r}$, momentum $\boldsymbol{p}$ and force $\boldsymbol{F}$ ) we define two pseudovectors: the angular momentum $\boldsymbol{L}=\boldsymbol{r} \times \boldsymbol{p}$ and the torque $\boldsymbol{M}=\boldsymbol{r} \times \boldsymbol{F}$. And then we get the fundamental equation: $\boldsymbol{M}=\mathrm{d} \boldsymbol{L} / \mathrm{d} t$ (Rao, 2006). The two pseudovectors are consistently introduced for particles, particle systems, and rigid bodies. Analysis of a rigid body rotation around a fixed axis " $z$ " leads to the concept of a rigid body moment of inertia $I_{\mathrm{z}}$ and allows creating the algorithmic framework for solving direct and inverse problems of the rotation dynamics:

$$
\begin{gathered}
\underset{\frac{d}{d t}}{\vec{\varphi}} \underset{\int t}{\stackrel{\frac{d}{d t}}{\rightleftarrows}} \vec{\omega} \vec{\varepsilon}=\frac{\vec{M}}{I} \\
\int d t \int d t
\end{gathered}
$$

The framework shows differential-integral relations of the angular characteristics $(\varphi, \omega$, $\varepsilon$ ) and the law of rotational dynamics (the Rotational Dynamics Equation).

Models prediction. With the help of the formed mathematical model the students are invited to analyze physical situations when the torque $\boldsymbol{M}$ is always perpendicular to the angular momentum $\boldsymbol{L}$ of a rotation rigid body. As a result of the model analysis, the students express the idea of a rigid body precessional movement.

Models verification and validation. The real gyro precession experiments are demonstrated to the students for the confirmation of the model's prediction. The students explain the gyroscope motion features using the developed model.

So in the context of the CTLPh cycle, the lecture demonstrated the working scientific knowledge cycle: facts $\rightarrow$ hypotheses $\rightarrow$ model $\rightarrow$ prediction $\rightarrow$ experiment $\rightarrow$ facts.

At the end of this lecture, analogy of the two concept models of a rigid body dynamics is demonstrated - the models of translational and rotational motions. And the two algorithmic frameworks (rotational and translational motion) are compared.

\subsection{Seminar "Dynamics of a rigid body rotational motion"}

The concepts, models and algorithms presented at the lecture "Dynamics of a rigid body rotational motion" are used at the seminar to solve physical problems.

The direct and inverse problems of rotation dynamics are considering. In the process of solving problems, the students have to find torques and moments of inertia of rigid bodies, to integrate the equations of motion. In the problems of blocks' rotating the students use the two algorithm frameworks together - for rotational and translational motion of rigid bodies.

Some examples of problems solved at the seminar:

- Calculation of a circular cone's moment of inertia. The calculation algorithm based on the moment of inertia additivity is discussed. The students offer two ways of splitting a right circular cone of uniform density into components and calculate the moment of inertia in two ways by integration. The students analyze and interpret the features of the resulting formula for the cone moment of inertia.

- Calculation of the Oberbeck pendulum moment of inertia. The students express the pendulum moment of inertia as the sum of the moments of inertia of several cylindrical bodies. The Huygens-Steiner theorem is used (Rao, 2006).

- Modeling of the Oberbeck pendulum rotation under the tension force torque of a thread wound on the pendulum pulley, with a load fixed on the thread free end. For solving dynamic problems the students use the two algorithmic frameworks together and kinematic relations of angular and linear characteristics of motion. 
The obtained dependences of the pendulum angular acceleration and the load lowering time are analyzed depending on the load mass and on the pendulum moment of inertia. The results are subject to experimental verification when the students perform lab works.

The CT components are contextually present in solving physical problems: abstraction, decomposition, analogy, algorithmization, generalization. The corresponding CT components are highlighted and emphasized in the process of solving problems.

\subsection{Laboratory work "Dynamics of a rigid body rotational motion"}

The goal of the laboratory work is testing theoretical representations and models of a rigid body rotational motion dynamics.

The solvable tasks for the students:

1. Experimental determination of the Oberbeck pendulum angular acceleration dependence on the external torque. Checking the basic law of a rigid body rotational motion.

2. Experimental determination of the Oberbeck pendulum moment of inertia dependence on the distribution of its mass relative to the axis of rotation. Verifying the additivity of a moment of inertia and its quadratic dependence on the distance to the rotation axis.

\subsection{Computer modeling "Dynamics of a rigid body rotational motion"}

As an example, we consider the virtual lab work "Precession and nutation of a gyroscope". The gyroscope experiment of 3B Scientific Corporation served as a real prototype for the student software project.

Previously, the students got acquainted with the phenomenon of precession and nutation of a gyroscope (Butikov, 2006).

To obtain three differential equations of motion, the students used the Lagrange method, having independently mastered it using literary sources. The Runge-Kutta method was used to numerically integrate the differentia equations of the gyroscope motion.

The software product was developed in the object-oriented programming language $\mathrm{C}++$. The 3D model of the virtual gyroscope was implemented in the Blender 3D graphics editor. The software graphics and interface were implemented using the Qt framework.

The main GUI window of the students' virtual lab (see Fig. 2) contains: the main menu bar, the gyroscope 3D animated image, the control elements (buttons, sliders), and the timer.

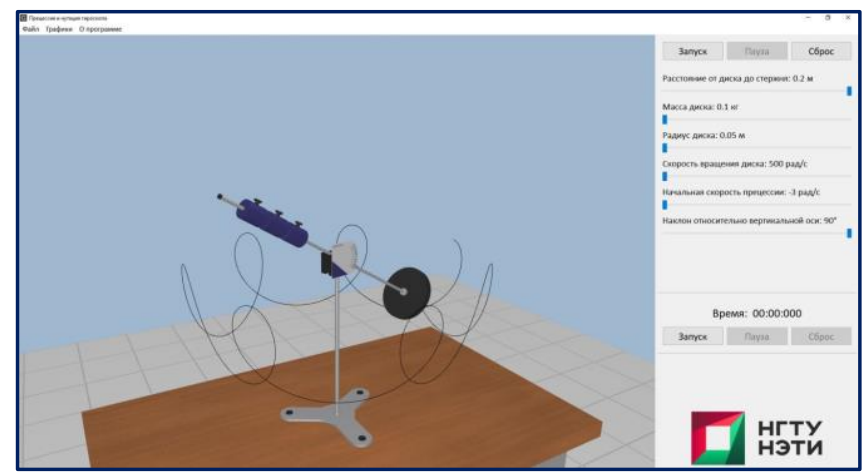

Fig.2. Virtual lab "Precession and nutation of a gyroscope" 
The program interface allows you to change the main parameters of the gyroscope: the distance to the disk, the mass and radius of the disk, the angular velocity of the disk rotation, the inclination of the gyroscope axis. The "Start", "Pause" and "Reset" buttons start and pause the process, reset the gyroscope parameters. The virtual timer makes it possible to determine the current time.

The gyroscope complicated movement is dynamically displayed in the main GUI window and is additionally accompanied by the image of the gyroscopic axis end's trajectory.

The program interface includes additional windows with a splash screen, theoretical help, user manual, information about project developers and function graphics.

This students' software product can be used as a virtual laboratory work and as an interactive virtual lecture demonstration.

\section{Conclusion}

As the author's pedagogical experiment, the CTLPh technology is used at the Faculty of Applied Mathematics and Computer Science of the NSTU NETI since 2010. The total number of students in the experimental groups was more than 400 people.

In the process of interviews, questionnaires, testing, seminars, laboratory studies, computer modeling and exams, IT students of the experimental groups demonstrated:

- a higher level of understanding of theoretical concepts, model concepts, the use of abstraction and idealization in physics;

- a higher degree of ability to use the concepts of modeling, abstraction, idealization, decomposition in solving problems of physics;

- a more advanced degree of using analogies, generalizations, and algorithms in distinguishing classes of physics problems in the process of their analysis and solution;

- a high ability to team-solve problems of computer modeling of physical processes and the creation of high-quality software products - interactive virtual physical laboratories.

During our pedagogical experiment, the IT students' teams created about 150 software products. Most of them are interactive virtual lab works with dynamic 3D visualization of simulated physical systems and processes. In the spring semester of 2019, the eight students' teams implemented the comprehensive IT project - an interactive virtual laboratory "Oscillators", combining eight different lab works.

Over the years of the author's pedagogical experiment, the IT students' teams independently organized and held four conferences with reports and demonstrations of their developments. The students' reports and software developments were highly appreciated by university professors and experts.

Some examples of student software developments are presented in the author's publications (Baranov, 2016, 2017, 2018).

According to the NSTU NETI professors and students, the author's CTLPh technology has demonstrated its effectiveness in the formation of professional thinking and competencies of the IT students in teaching physics at the Technical University.

\section{References}

1. A.V. Aho, Computation and Computational Thinking, in The Computer Journal, 55(7), pp. 832-835 (2012). DOI: 10.1093/comjnl/bxs074

2. A.V. Baranov, Computer modelling in the Physics course for IT students, in Computer Modelling and New Technologies, 21(3), pp. 45-49, (2017) 
3. A.V. Baranov, Virtual Students' Laboratories in the Physics Practicum of the Technical University, In Proceedings of the13th International Scientific-Technical Conference on Actual Problems of Electronics Instrument Engineering (APEIE), Vol.1, pp. 326-328, Novosibirsk: NSTU NETI (2016). DOI: $10.1109 /$ APEIE.2016.7802287

4. A.V. Baranov, Students' project developments of wave optics virtual labs, In Proceedings of the14th International Scientific-Technical Conference on Actual Problems of Electronics Instrument Engineering (APEIE), Vol.1, pp. 240-242, Novosibirsk: NSTU NETI (2018). DOI: 10.1109/APEIE.2018.8545025

5. E. Butikov, Precession and nutation of a gyroscope, in European Journal of Physics, 27(5), pp. 1071 - 1081 (2006). DOI:10.1088/0143-0807/27/5/006

6. R. Chabay \& B. Sherwood, Computational physics in the introductory calculusbased course, in Am. J. Phys, 76(4\&5), pp. 307-313 (2008). DOI: $10.1119 / 1.2835054$

7. C.C. Chea, J. T. J. Huan, Higher Education 4.0: The Possibilities and Challenges, in Journal of Social Sciences and Humanities, 5(2), pp. 81-85 (2019). http://www.aiscience.org/journal/jssh

8. T. De Jong, M.C. Linn, C.Z. Zacharia, Physical and Virtual Laboratories in Science and Engineering Education, in Science, 340 (19 April), pp. 305-308 (2013)

9. P.J. Denning, Remaining Trouble Spots with Computational Thinking, in Communications of the ACM, 60(6), pp. 33-39 (2017). DOI:10.1145/2998438

10. A. DiSessa, Changing Minds: Computers, in Learning and Literacy, Cambridge, MA: MIT Press (2000)

11. A.P. Ershov, Programming, the second literacy, in Microprocessing and Microprogramming, 8(1), pp. 1-9 (1981). https://doi.org/10.1016/01656074(81)90002-8

12. E. Etkina, A. Warren \& M. Gentile, The Role of Models in Physics Instruction, in The Physics Teacher, 44(1), pp. 34-39 (2006). DOI: 10.1119/1.2150757

13. N. Finkelstein, Learning Physics in Context: A study of student learning about electricity and magnetism, in International Journal of Science Education, 27(10), pp. 1187-1209 (2005). DOI: 10.1080/09500690500069491

14. H. Gould, J. Tobochnik, Integrating Computation into the Physics Curriculum, In Alexandrov V.N., Dongarra J.J., Juliano B.A., Renner R.S., Tan C.J.K. (eds) Computational Science - ICCS 2001. ICCS 2001. Lecture Notes in Computer Science, vol 2073, Springer, Berlin, Heidelberg, pp. 1031-1040 (2001)

15. I.A. Halloun, Modeling Theory in Science Education, Berlin: Springer (2004)

16. D. Hestenes, Notes for a Modeling Theory of Science, Cognition and Instruction, in Proceedings of the 2006 GIREP conference: Modelling in Physics and Physics Education, August 20 - 25, Amsterdam, Netherlands, pp. 34-65 (2006)

17. T-C. Hsu, S-C. Chang, Y-T. Hung, How to learn and how to teach computational thinking: Suggestions based on a review of the literature, in Computers \& Educations, 126, pp. 296-310 2018. https://doi.org/10.1016/j.compedu.2018.07.004

18. P. Johnson-Laird, Mental models, Cambridge: Harvard University Press, (1983).

19. D.A. Kolb, Individual learning styles and the learning process, Cambridge: Massachusetts Institute of Technology, Sloan School of Management, pp. 535-571 (1971)

20. D.A. Kolb, Experiential learning: Experience as the Source of Learning and Development, 2nd edition, Pearson Education, New Jersey (2015)

21. R. Landau, Computational Physics: A Better Model for Physics Education?, in Computing in Science \& Engineering, 8(5), pp. 50-58 (2006). DOI: $10.1109 /$ MCSE.2006.85 
22. E.A. Lee, The Past, Present and Future of Cyber-Physical Systems: A Focus on Models, in Sensors, 15(3), pp. $4837-4869$ (2015). DOI:10.3390/s150304837

23. Merriam-Webster, On-Line Dictionary (2019). http://www.webster.com

24. H.A. Müller, Rise of Intelligent Cyber-Physical Systems, in Computer, 50(12), pp. 7-9 (2017). DOI: 10.1109/MC.2017.4451221

25. S. Papert, Mindstorms: Children, Computers, and Powerful Ideas, in NY: Basic Books, Inc., New York, (1980)

26. M. Prensky, Students as designers and creators of educational computer games: Who else?, in British Journal of Educational Technology, 39(6), pp. 1004-1019 (2008) DOI:10.1111/j.1467-8535.2008.00823_2.x

27. A. Rao, Dynamics of particles and rigid bodies: a systematic approach, in Cambridge University Press (2006)

28. E.F. Redish, Millikan Award Lecture (1998): Building a Science of Teaching Physics, in The American Journal of Physics, 67, pp. 562-573 (1999). https://doi.org/10.1119/1.19326

29. L.G. Rimoldini, C. Singh, Student understanding of rotational and rolling motion concepts, in Phys. Rev. St Phys. Educ. Res., 1, 010102, pp. 1-9 (2005). DOI: 10.1103/PhysRevSTPER.1.010102

30. K. Schwab, The Fourth Industrial Revolution, London: Penguin Random House.

31. J.A. Stankovic, J.W. Sturges, J. Eisenberg, A 21st Century Cyber-Physical Systems Education, in Computer, 50(12), pp. 82-85, (2017). DOI:10.1109/MC.2017.4451222

32. M. Tedre, P.J. Denning, The Long Quest for Computational Thinking. Proceedings of the 16th Koli Calling Conference on Computing Education Research, November 2427 2016, Koli, Finland: pp. 120-129 (2016). DOI: http://dx.doi.org/10.1145/2999541.2999542

33. A.A. Verbitsky, V.G. Kalashnikov, Contextual Approach in Psychology, in European Scientific Journal, 9(32), pp. 1-12 (2013)

34. T. Wallner, G. Wagner, Academic Education 4.0. Proceedings of the END 2016 International Conference on Education and New Developments, June 12-14 2016, Ljubljana, Slovenia, pp. 155-159 (2016)

35. J.M. Wilson, E.F. Redish, Using Computers in Teaching Physics, in Physics Today, 42(1), pp. 34-41 (1989). https://doi.org/10.1063/1.881202

36. J.M. Wing, Computational Thinking, in Communications of the ACM, 49(3), pp. 3335, (2006). https://doi.org/10.1145/1118178.1118215

37. B.M. Zwickl, D. Hu, N. Finkelstein, H.J. Lewandowski, Model-based reasoning in the physics laboratory: Framework and initial results, in Phys. Rev. St Phys. Educ. Res, 11, 020113 (2015). DOI: 10.1103/PhysRevSTPER.11.020113 